\title{
Low Cost Humidity Sensor Based on PANI/PEDOT:PSS Printed on Paper
}

\author{
Rogério M. Morais, Maykel dos Santos Klem ${ }^{(}{ }^{\infty}$, Gabriel Leonardo Nogueira, \\ Tiago Carneiro Gomes, and Neri Alves
}

\begin{abstract}
The production of low cost sensors to monitor environment in some industrial sectors is a current need. In aviculture centers, for example, the concentration of ammonia gas is related to humidity, and it is necessary to control it to avoid contamination. With this need in mind, this paper presents the preparation and characterization of a low cost humidity sensor based on poly(3,4-ethylenedioxythiophene)-poly(styrene sulfonate) and polyaniline fully printed onto paper by a commercial HP printer. Using electrochemical impedance spectroscopy, an equivalent circuit containing resistive and capacitive parameters was proposed. The resistive parameter $\mathrm{R} 1$ is related to values of ambient humidity. The $R 1$ values showed a sensitive response of $200 \%$ when relative humidity changes $80 \%$, taking $10 \mathrm{~min}$ to reach saturation point. Moreover, the device showed good stability when humidity remained constant. These results indicate that the proposed device is suitable for applying as a humidity sensor that costs less than 1 Euro cent.
\end{abstract}

Index Terms-Ink jet printing, PANI, paper based device, PEDOT:PSS, printed humidity sensor.

\section{INTRODUCTION}

$\mathbf{M}$ ONITORING and controlling the environment is highly important in many industrial sectors, for example, food, paper and aviculture. In the food industry, there is a need to control temperature, humidity and light during storage and transport to guarantee quality and extend lifetime. During paper production, humidity control is necessary to ensure the product meets weight specifications. In aviculture centers, the concentration of ammonia gas is directly linked to humidity so controlling it is necessary to avoid contaminations of animals and workers [1]. The cost of this type of monitoring should be kept as low as possible in order not to affect the final price of the product. Against this background, interest in low-cost devices to monitor humidity, qualitatively or quantitatively, has increased recently [2]-[8]. The category of materials that serves this need are conductive polymers. They enable the production of circuits and electronic devices on a variety of

Manuscript received November 28, 2017; revised January 16, 2018; accepted January 21, 2018. Date of publication February 6, 2018; date of current version March 9, 2018. This work was supported in part by the Programa de Pós-graduação em Ciência e Tecnologia de Materiais, in part by the Coordenação de Aperfeiçoamento de Pessoal de Nível Superior, and in part by the Fundação de Amparo à Pesquisa do Estado de São Paulo. The associate editor coordinating the review of this paper and approving it for publication was Dr. Camilla Baratto. (Corresponding author: Maykel dos Santos Klem.)

The authors are with the School of Technology and Sciences, São Paulo State University (UNESP), Presidente Prudente SP 19060-900, Brazil (e-mail: maykel_klem@live.com).

Digital Object Identifier 10.1109/JSEN.2018.2803018 substrates at room temperature, with low cost production and good performance [2], [9]-[11].

Polymers poly(3,4-ethylenedioxythiophene)-poly(styrene sulfonate) (PEDOT:PSS) [11] and polyaniline (PANI) [12] are widely used in organic devices because of their relatively low cost, solubility, and easy handling. PEDOT:PSS can be used as an active layer in various types of sensor [13], [14], but, due to its relatively high conductivity, it is most frequently used as the electrode in organic devices [16]-[19]. Besides, its conductivity can be enhanced by more than two orders of magnitude by adding certain organic solvent such as ethylene glycol [19], [20]. Among other organic polymers used in humidity sensors, PANI has become the object of studies because the water absorption process is directly related to the formation of protons that dope the polymer increasing its conductivity [13], [20].

The use of PANI and PEDOT:PSS together have already been reported, for example, in the electrochromic device by Delongchamp and Hammond [22], and in a transparent and flexible electrode tested in a pH sensor by Vacca et al. [23]. Another important point of Vacca et al work, is the deposition of PEDOT:PSS by inkjet printing, which is a low-cost deposition technique compatible with flexible substrates, like polyethylene terephthalate (PET), paper, and others. Paper stands out in production of low cost devices, being attractive for application as conventional substrate or active element in organic electronics [21]-[25]. It is mainly constituted of cellulose, and shows various advantages and interesting characteristics including low cost, abundance, flexibility, availability in different types, biodegradability, chemical and thermal stability, and strong adhesion to many materials [29].

Herein, we present the electrical characterization of a low cost (see Fig. 1.) humidity sensor composed of PANI as the sensing material and PEDOT:PSS as the electrodes, both printed onto paper by a commercial inkjet printer.

\section{Details OF EXPERIMENTS}

\section{A. Ink Jet Printer}

A Hewlett-Packard $®(\mathrm{HP})$ commercial inkjet printer, model J110a, was used to print polymeric inks of PANI and PEDOT:PSS onto bond paper (BP). The original cartridge was opened and the ink, sponge and internal filter were removed. In order to eliminate all commercial ink residues, a careful cleansing was performed using 2-propanol 
Experimental setup and production cost of sensors

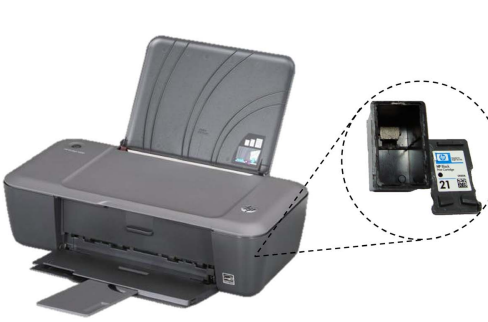

Office inkjet printer cost: $€ 50$

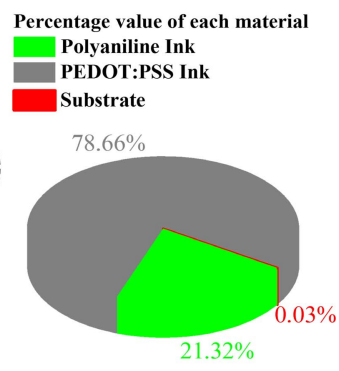

Sensor cost: $€ 0.009$
Fig. 1. Image of an inkjet printer and its cartridge used in this study. Percentage values of each material used to produce the sensor are also presented.

alcohol $((\mathrm{CH} 3) 2 \mathrm{CHOH})$ and deionized water. Fig. 1. shows a schematic of low cost system including a commercial printer picture and a pie chart showing percentage value of each material used in sensor fabrication. The printing system costs about $€ 50$, and each sensor less than 1 euro cent.

\section{B. Ink Formulation and Printing of PANI and PEDOT:PSS}

Polyaniline, used as bought from Sigma Aldrich, was add to 1-Methyl-2-pyrrolidinone $(10 \mathrm{mg} / \mathrm{ml})$ and stirred for 4 hours. The solution obtained was added to ethylene glycol and ultrapure water (Milli-Q) in 1:1:2 (v:v:v) proportion, then stirred for two more hours. This final solution was passed through a Millex HV PVDF $0.45 \mu \mathrm{m}$ filter to remove any remaining particles. The PEDOT:PSS based ink was prepared adding this polymer (1.3\% wt dispersion in water - Sigma-Aldrich) to ethylene glycol and ultrapure water in the following proportion 2:1:1 (v:v:v). The solution was stirred for 2 hours and filtered with a Millex filter. PANI and PEDOT:PSS inks were analyzed by a Brookfield DV1 viscometer in room conditions, showing viscosity of 4.49 and $6.5 \mathrm{cP}$ respectively.

The geometric patterns of active layer (PANI) and electrodes (PEDOT:PSS) were designed in Microsoft Word®software. The printing number (PN) of PANI in the same position was varied from 1 to 5 and this number was kept constant at 5 for the PEDOT:PSS electrodes. The maximum tonality provided by the printer system was used to print the active layer and electrodes. The samples were prepared by printing the polymeric inks onto commercial bond paper of $75 \mathrm{gm}^{-2}$ density and without any previous treatment. The dimensions are shown in Fig. 2.a.

\section{Sample Doping Process}

The samples containing Polyaniline need to pass through a doping process, because polyaniline in an emeraldine base form behaves like an insulating material. The samples were therefore exposed to $\mathrm{HCl}$ (1 M - Sigma-Aldrich) vapor for 40 minutes in a closed recipient. After this, the color of active layer changed from blue (emeraldine base) to green (emeraldine salt), indicating that the PANI had reached a conductive state. Before electric characterization, the samples were left
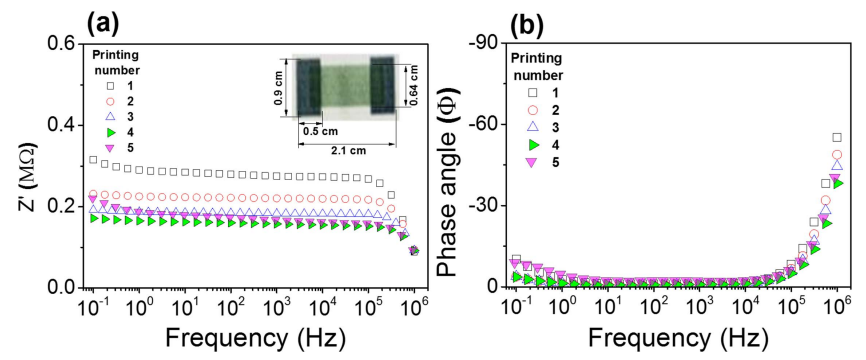

Fig. 2. (a) Real impedance and (b) phase angle plots for variations in the PN number of PANI layers. Inset shows a digital picture of a printed device.

exposed to air for at least 4 hours at room temperature, to evaporate the excess of $\mathrm{HCl}$.

\section{Electrical Characterization and Experimental Set-Up}

Keithley source meter model 2420, was used for electrical measurements. An impedance analyzer Solartron 1260 with dielectric interface 1296 was used for electrochemical impedance spectra (EIS) measurements. A homemade system for humidity control was used to characterize the samples in different relative percentages of humidity. This system is composed of a chamber with a small amount of water inside kept in thermal contact with an external water flow in which the temperature is controlled. Different values of relative humidity can be obtained based on the temperature difference between the water inside the chamber and the region where the sample is located [30]. An Incotherm ${ }^{\circledR}$ thermo-hygrometer was used to monitor the humidity at each measurement.

\section{RESUlts AND Discussions}

\section{A. The Device's Electrical Characterization}

The Bode plot for changes in PN from 1 to 5 is showed in Fig. 2.a. The measurements were realized in room conditions (RC), with a temperature of $24{ }^{\circ} \mathrm{C}$ and $40 \%$ relative humidity. When PN increases, an improvement in film uniformity is observed and, naturally, its thickness increases. This can be shown by the reduction of Z' by half when PN increases.

Phase angle analyses were taken to investigate the resistive/ capacitive behavior of the devices, as seen in Fig. 2.b. Through this measurement, it is possible to determine the frequency range where the device exhibits a resistive $(\Phi=0)$ or capacitive $(\Phi=-90)$ behavior. A small increase in phase angle for frequencies lower than $1 \mathrm{~Hz}$ was observed. However, by increasing PN, phase angle reduces due to an increase in electrical conductivity. A predominantly resistive regime extending from approximately $5 \mathrm{~Hz}$ to $10^{4} \mathrm{~Hz}$ was also observed. Therefore, at this frequency range, all the samples can be seen to be acting as a resistor, independent of printing number. Then, for a practical viewpoint, the values of impedance for each PN are acceptably close. Due to this fact, and trough empirical experimentation, $\mathrm{PN}=3$ was used for PANI in all measurements shown below. In other words, devices aiming humidity sensor applications were fabricated using PN $=3$ for printing PANI films because: 
(a)
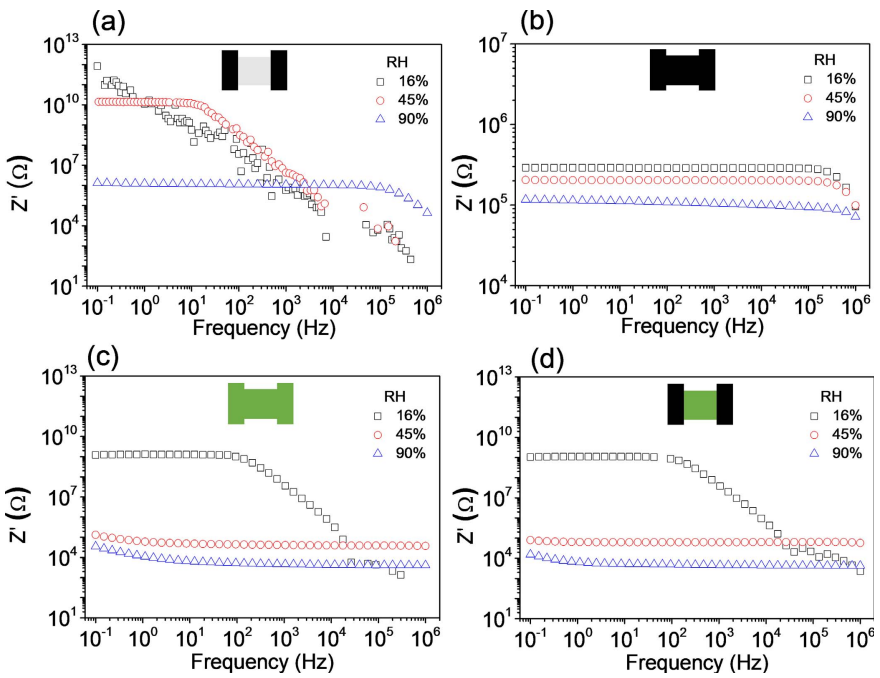

Fig. 3. Real impedance versus frequency curves for (a) uncovered bond paper with small PEDOT:PSS rectangles to act as electrical contact; (b) PEDOT:PSS; (c) PANI films; and (d) the main device consisted of PANI and PEDOT:PSS printed onto paper.

i) the resistivity with $\mathrm{PN}=3$ is not so high; ii) films produced with $\mathrm{PN}=3$ show a predominance of the resistive regime in a broad frequency range, similar to those with larger PN; and iii) fabrication route becomes simpler, since higher printing numbers do not produce any significant improvements.

\section{B. Interaction With Humidity}

Measures of EIS were taken to evaluate how each material interacts with humidity. The samples were exposed at low, medium and high relative humidity conditions with values of $90 \%, 45 \%$ and $16 \%$, respectively. Fig. 3 shows real impedance versus frequency curves for the following samples: uncovered bond paper (Fig. 3.a) between PEDOT:PSS pads; PEDOT:PSS (Fig. 3.b) and PANI (Fig. 3.c) films printed onto paper; and the device itself (Fig. 3.d) consisting of PANI and PEDOT:PSS. Proceeding as in the main device set-up, PANI films were printed three times at the same position, and PEDOT:PSS five times.

A reduction in real impedance was observed when the measurements are taken in high humidity for all devices configurations. In high relative humidity, the PANI film exhibits real impedance values two orders of magnitude lower than pure bond paper. With the presence of water, protonation occurs in nitrogen atoms. This effect results in the formation of stabilized polycations between $\pi$ delocalization along the polymeric chain, enhancing electrical conductivity [28]. Among the four samples of Fig. 3, PEDOT:PSS film printed onto paper (Fig. 3.b) showed the smaller impedance variation when $\mathrm{RH}$ changing from $16 \%$ to $90 \%$, lower than one order of magnitude. The electrical behavior of sensors (Fig. 3.d) with humidity changes resembles the samples containing only PANI (Fig. 3c). It can therefore be said that impedance value is directly related to water absorption in the PANI layer, and the printed PEDOT:PSS acts only like a conductive electrode.

Nyquist diagram obtained from different relative humidity values, ranging from 22 to $90 \%$, is shown in Fig 4.a.
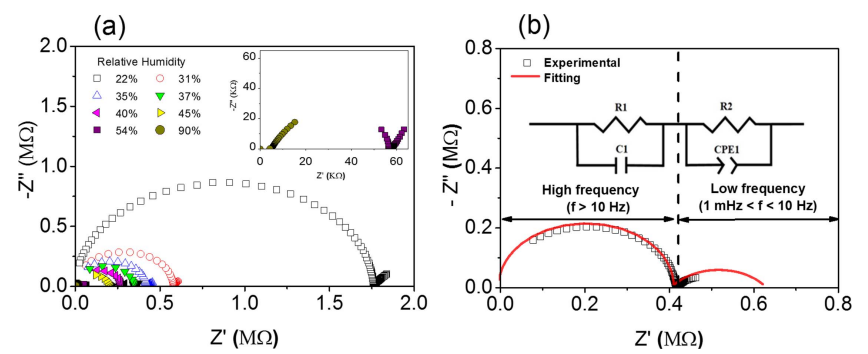

Fig. 4. (a) Nyquist diagram for samples in different environment humidity exposure. Figure inset shows the impedance curves for relative humidity of $51 \%$ and $90 \%$. Figure 4.b. shows the Nyquist diagram with the EIS data fitting. Figure inset presents the equivalent circuit obtained.

TABLE I

Parameter Values ObTained From the is Data

\begin{tabular}{l|r}
\hline \hline Parameters & Values \\
\hline \hline Resistance (R1) & $0.41 \mathrm{M} \Omega$ \\
\hline Capacitor (C1) & $0.78 \mathrm{pF}$ \\
\hline Resistance (R2) & $0.21 \mathrm{M} \Omega$ \\
\hline $\mathrm{Q}$ & $22.41 \mu \mathrm{s} / \Omega$ \\
\hline $\mathrm{n}$ & 0.57 \\
\hline Chi - Square & 0.001 \\
\hline
\end{tabular}

The semicircles observed characterize two different polarization processes and can be represented by an equivalent circuit containing two RCs branches. Each semicircle predominates at different frequency ranges. Fig. 4.b. shows a curve modelling for the Nyquist diagram relative to RH equal 35\%, obtained from Zview ${ }^{\circledR}$ software.

At low frequencies $(0.1 \mathrm{~Hz}-10 \mathrm{~Hz})$, a resistor $\mathrm{R} 2$ and a constant phase element (CPE) represent the first semicircle. At higher frequencies $(>10 \mathrm{~Hz})$, a second semicircle is represented by a resistor $\mathrm{R} 1$ and a capacitor $\mathrm{C} 1$. It is worth to emphasize that the equivalent circuit in low frequency region might change from one batch of samples to another. But in high frequency region the impedance behavior is always the same, allowing to model all curves showed in Fig. 4.a. However, as the value of $\mathrm{C} 1$ is very low $(\sim 0.8 \mathrm{pF})$, it is thought that its origin resides in the measure system. The right branch is described by resistance coupled with a constant phase element (CPE), giving by $z=1 / Q(j \omega)^{n}$. A CPE is related to a distribution of time constants, which occurs mainly in irregular and porous electrodes [31]. All parameters used to model the curve are show in Tab. 1.

Fig. 5 shows the parameters obtained from Nyquist diagram (Fig. 4.a) modelling plotted against humidity variation. As seen in Fig. 5.a and 5.b, parameters Q, n, C1 e R2, have a weak match within $\mathrm{RH}$ variations. The main parameter related to environmental humidity, showed in Fig. 5.c., is the value of equivalent series resistance R1, which can be considered a series resistance because $\mathrm{C} 1$, a capacitance from the system, has to be disregarded. The water molecules are trapped by the substrate due to the hygroscopic property of paper [26]. In association with acid ions remaining from the doping process, a sort of electrolyte is formed causing a resistance drop when an electric field is applied. 

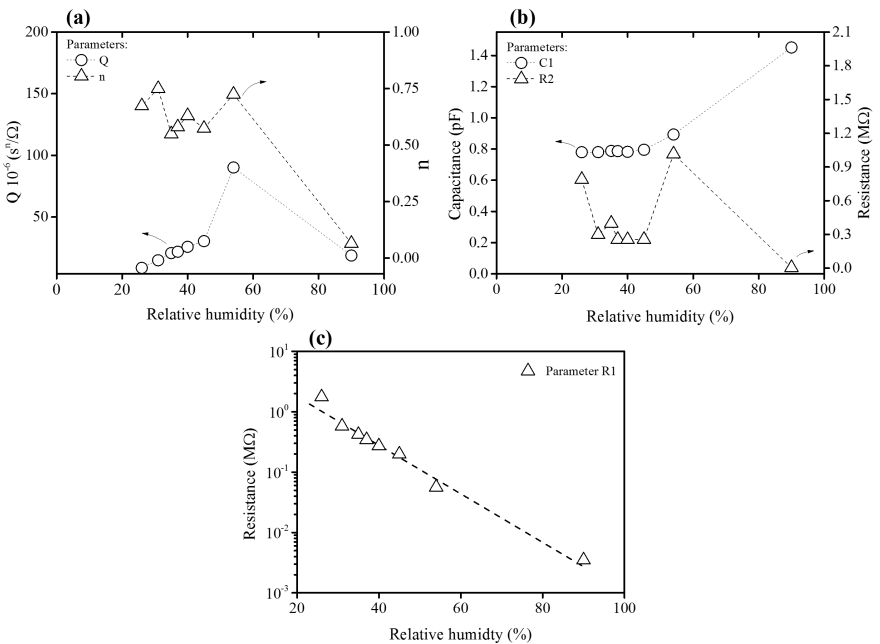

Fig. 5. Values of (a) Q, n, (b) R2, C1 and c) R1 parameters obtained from the equivalent circuit fitting of EIS measurements in different relative humidity exposure.

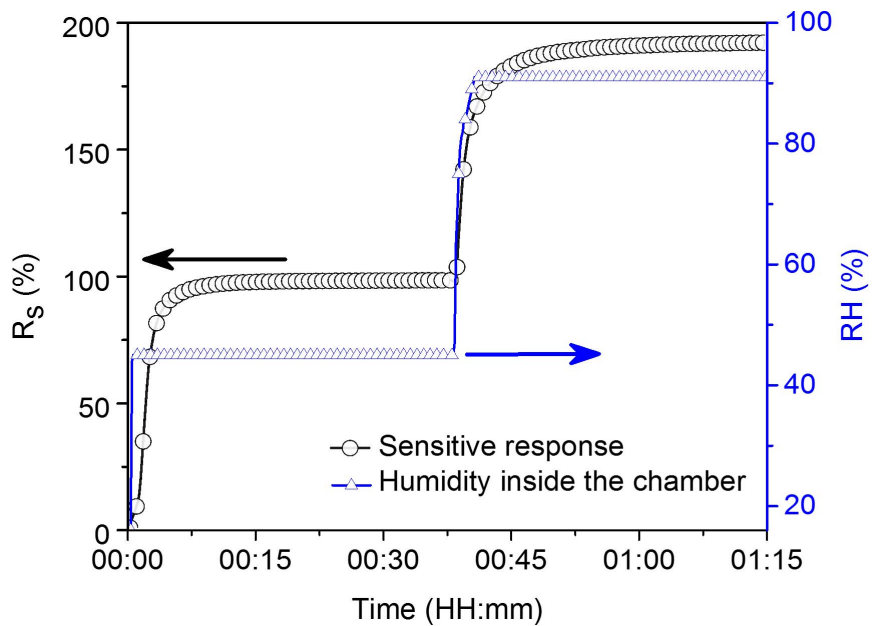

Fig. 6. Sensitive response during periods in which the humidity was kept constant at two different values ( $45 \%$ and $90 \%$ ), and the humidity variation inside the chamber.

\section{Sensitive Response and Response Time}

EIS was used at a constant frequency $\left(10^{3} \mathrm{~Hz}\right)$ to measure sensor response and response time. The sensitive response was taken from the equation $R_{S}=\left[\left(Z_{0}-Z_{1}\right) / Z_{1} \times 100\right]$, where $\mathrm{Z}_{0}$ is the impedance module before increasing humidity in the system, and $\mathrm{Z}_{1}$ the impedance module at the instant of measurement. The response time was obtained when the relative humidity changed from $16 \%$ to $45 \%$ and from $45 \%$ to $90 \%$.

The sensitive response during periods in which the humidity was kept constant at two different values (45\% and 90\%) is shown in Fig.6. When the relative humidity increases from $16 \%$ to $45 \%$, the device exhibits a sensitive response of $100 \%$. The impedance values experienced variations of nearly $100 \%$, taking 9 minutes to achieve the saturation state. When humidity variation increases from $45 \%$ to $90 \%$, the sensitive response is also $100 \%$, taking 12 minutes to reach the saturation state.

Results shown in Fig. 6 were obtained by EIS measurements. However, as previously discussed, it is sufficient to determine the resistance R1. To find this parameter, voltage
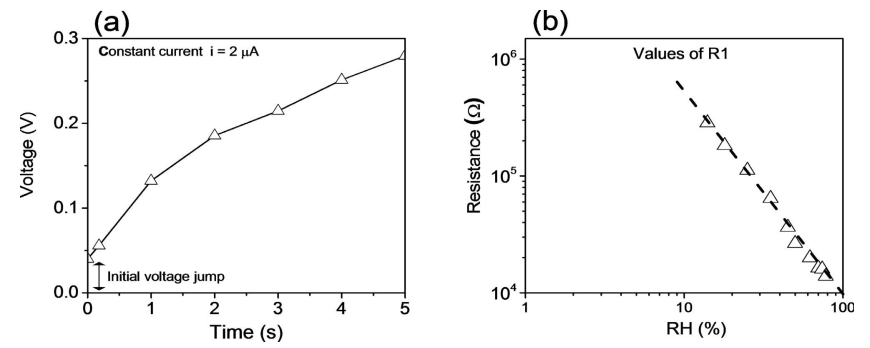

Fig. 7. (a) Vxt curve for a sample submitted to a constant current of $2 \mu \mathrm{A}$. Detail shows the initial voltage jump where R1 can be extracted. (b) R1 values versus humidity displayed in logarithmic scale obtained by the constant current technique.

versus time (Vxt) can be measured keeping the electric current constant across the sample. With this alternative, the $\mathrm{R} 1$ value can be measured in a few tenths of a second. In this approach, the voltage is applied for a short time, avoiding the degradation of the device. The Vxt characterization for a sensor subjected to a constant current of $2 \mu \mathrm{A}$ is shown in Fig. 7.a. The R1 value was then obtained from the initial voltage jump $(\mathrm{t} \sim 3 \mathrm{~ms})$. This value, measured at the beginning of the Vxt curve, corresponds to those at high frequencies in the EIS. R1 values versus humidity obtained by this constant current technique are plotted in Fig. 7.b. A linear variation in a logarithmic scale for R1 values can be observed as function of relative humidity.

Both techniques, EIS and Vxt, were employed to characterize the as-proposed humidity sensor. Therefore, constant current technique is compatible with the low cost proposal. With some operational amplifiers and an arduino®controller, it is possible to build a simple electrical system that applies a constant current and measure the correspondent voltage, that will cost less than a couple of tens euros. This fact matches with the low cost to produce the sample depicted in Fig. 1.

\section{CONCLUSION}

In this paper the production of a low cost humidity sensor based in PANI/PEDOT:PSS printed onto paper using a commercial printer has been presented. A reduction of several orders of magnitude in resistance values for PANI films printed onto paper was observed as the printing number $(\mathrm{PN})$ increased. An equivalent circuit representing the device was proposed, containing the parameters R1, R2 and CPE. We show that the parameter R1 gives a better relation (linear $\log$-log scale) within humidity variations. The device's sensitive response is $200 \%$ when there is a humidity change from $16 \%$ to $98 \%$ and it takes about 10 min to reach the saturation state. Constant current measurements show it to be a good alternative for obtaining variations of R1 with good accuracy, fast response and avoiding sensor degradation.

\section{REFERENCES}

[1] Y. S. Chen, Y. Li, and M. J. Yang, "A fast response resistive thin film humidity sensor based on poly(4-vinylpyridine) and poly(glycidyl methacrylate)," J. Appl. Polym. Sci., vol. 105, no. 6, pp. 3470-3475, 2007.

[2] Y. Li, C. Deng, and M. Yang, "A novel surface acoustic wave-impedance humidity sensor based on the composite of polyaniline and poly(vinyl alcohol) with a capability of detecting low humidity," Sens. Actuators B, Chem., vol. 165, no. 1, pp. 7-12, Apr. 2012. 
[3] K. Crowley, M. Smyth, A. Killard, and A. Morrin, "Printing polyaniline for sensor applications," Chem. Papers, vol. 67, no. 8, pp. 771-780, Dec. 2013.

[4] V. V. Chabukswar, S. Pethkar, and A. A. Athawale, "Acrylic acid doped polyaniline as an ammonia sensor," Sens. Actuators B, Chem., vol. 77, no. 3, pp. 657-663, 2001

[5] L. Huang et al., "A novel paper-based flexible ammonia gas sensor via silver and SWNT-PABS inkjet printing," Sens. Actuators B, Chem., vol. 197, pp. 308-313, Jul. 2014.

[6] D. Nilsson, T. Kugler, P. O. Svensson, and M. Berggren, "An all-organic sensor-transistor based on a novel electrochemical transducer concept printed electrochemical sensors on paper," Sens. Actuators B, Chem. vol. 86, nos. 2-3, pp. 193-197, 2002.

[7] P.-G. Su and C.-S. Wang, "Novel flexible resistive-type humidity sensor," Sens. Actuators B, Chem., vol. 123, no. 2, pp. 1071-1076, May 2007.

[8] H. Yan et al., "A high-mobility electron-transporting polymer for printed transistors," Nature, vol. 457, pp. 679-686, Feb. 2009

[9] A. Kokil, K. Yang, and J. Kumar, "Techniques for characterization of charge carrier mobility in organic semiconductors," J. Polym. Sci., B, Polym. Phys., vol. 50, no. 15, pp. 1130-1144, Aug. 2012.

[10] H. E. Katz and J. Huang, "Thin-film organic electronic devices," Аnпи. Rev. Mater. Res., vol. 39, no. 1, pp. 71-92, 2009.

[11] W. Lövenich, "PEDOT-properties and applications," Polym. Sci. Ser. C, vol. 56, no. 1, pp. 135-143, 2014.

[12] A. G. MacDiarmid and A. J. Epstein, "Polyanilines: A novel class of conducting polymers," Faraday Discussions Chem. Soc., vol. 88, pp. 317-332, May 1989.

[13] Y. Seekaew, S. Lokavee, D. Phokharatkul, A. Wisitsoraat, T. Kerdcharoen, and C. Wongchoosuk, "Low-cost and flexible printed graphene-PEDOT:PSS gas sensor for ammonia detection," Org. Electron. Phys., Mater. Appl., vol. 15, no. 11, pp. 2971-2981, Nov. 2014.

[14] M. Borghetti, M. Serpelloni, E. Sardini, and S. Pandini, "Mechanical behavior of strain sensors based on PEDOT:PSS and silver nanoparticles inks deposited on polymer substrate by inkjet printing," Sens. Actuators A, Phys., vol. 243, pp. 71-80, Jun. 2016.

[15] J. G. Tait et al., "Spray coated high-conductivity PEDOT: PSS transparent electrodes for stretchable and mechanically-robust organic solar cells," Solar Energy Mater. Solar Cells, vol. 110, pp. 98-106, Mar. 2013.

[16] C. M. Palumbiny, F. Liu, T. P. Russell, A. Hexemer, C. Wang, and P. Müller-Buschbaum, "The crystallization of PEDOT: PSS polymeric electrodes probed in situ during printing," Adv. Mater., vol. 27, no. 22, pp. 3391-3397, 2015.

[17] N. K. Unsworth et al., "Highly conductive spray deposited poly (3, 4-ethylenedioxythiophene):poly (styrenesulfonate) electrodes for indium tin oxide-free small molecule organic photovoltaic devices," Appl. Phys. Lett., vol. 103, no. 17, p. 173304, 2013.

[18] B.-J. Kim, S.-H. Han, and J.-S. Park, "Sheet resistance, transmittance, and chromatic property of CNTs coated with PEDOT: PSS films for transparent electrodes of touch screen panels," Thin Solid Films, vol. 572, pp. 68-72, Dec. 2014.

[19] H. Okuzaki, Y. Harashina, and H. Yan, "Highly conductive PEDOT/PSS microfibers fabricated by wet-spinning and dip-treatment in ethylene glycol," Eur. Polym. J., vol. 45, no. 1, pp. 256-261, 2009.

[20] J. Ouyang, C.-W. Chu, F.-C. Chen, Q. Xu, and Y. Yang, "Highconductivity poly(3,4-ethylenedioxythiophene):poly(styrene sulfonate) film and its application in polymer optoelectronic devices," Adv. Funct. Mater, vol. 15, no. 2, pp. 203-208, 2005.

[21] S. Stafström et al., "Polaron lattice in highly conducting polyaniline: Theoretical and optical studies," Phys. Rev. Lett., vol. 59, no. 13, pp. 1464-1467, 1987.

[22] B. D. Delongchamp and P. T. Hammond, "Layer-by-layer assembly of PEDOT/polyaniline electrochromic devices," Adv. Mater., vol. 13, no. 19 , pp. $1455-1459,2001$.

[23] A. Vacca et al., "Preparation and characterisation of transparent and flexible PEDOT: PSS/PANI electrodes by ink-jet printing and electropolymerisation," RSC Adv., vol. 5, no. 97, pp. 79600-79606, 2015.

[24] Y.-H. Kim, D.-G. Moon, and J.-I. Han, "Organic TFT array on a paper substrate," IEEE Electron Device Lett., vol. 25, no. 10, pp. 702-704, Oct. 2004

[25] J. Kawahara, P. A. Ersman, K. Katoh, and M. Berggren, "Fast-switching printed organic electrochemical transistors including electronic vias through plastic and paper substrates," IEEE Trans. Electron Devices, vol. 60, no. 6, pp. 2052-2056, Jun. 2013.

[26] E. Fortunato, N. Correia, P. Barquinha, L. Pereira, G. Goncalves, and R. Martins, "High-performance flexible hybrid field-effect transistors based on cellulose fiber paper," IEEE Electron Device Lett., vol. 29, no. 9, pp. 988-990, Sep. 2008.
[27] F. Zabihi and M. Eslamian, "Characteristics of thin films fabricated by spray coating on rough and permeable paper substrates," J. Coatings Technol. Res., vol. 12, no. 3, pp. 489-503, 2015.

[28] C. Nash et al., "A comparative study on the conductive properties of coated and printed silver layers on a paper substrate," J. Electron. Mater, vol. 44, no. 1 , pp. 497-510, 2015.

[29] D. Tobjörk and R. Österbacka, "Paper electronics," Adv. Mater, vol. 23 , no. 17, pp. 1935-1961, May 2011.

[30] M. V. Kulkarni and A. K. Viswanath, "Spectroscopic, thermal and electrical properties of sulphonic acids doped poly(o-anisidine) and their application as humidity sensor," Sens. Actuators B, Chem., vol. 107, no. 2, pp. 791-797, Jun. 2005.

[31] D. S. Yoo, A. Mahmoudzadeh, E. C. W. Fok, K. Walus, and J. D. W. Madden, "Multiple time constant modelling of a printed conducting polymer electrode," Electrochim. Acta, vol. 56, no. 13, pp. 4711-4716, 2011.

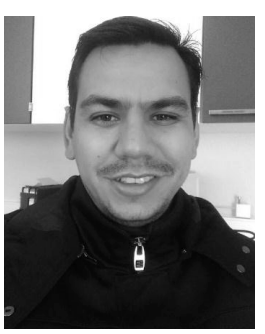

Rogério M. Morais was born in Presidente Prudente, Brazil, in 1985. He received the Licentiate degree in physics from the Universidade Estadual Paulista Júlio de Mesquita Filho in 2013, and the master's degree in materials science and technology in 2015 . He is currently pursuing the Ph.D. degree with the Post-Graduation Program in Materials Science and Technology (POSMAT). He has experience in physics, with emphasis on general physics, conductive polymers, organic electronics, printed electronics, and materials engineering.

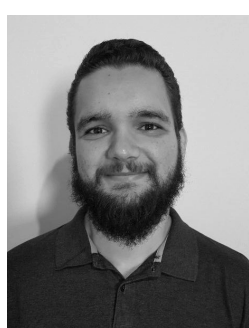

Maykel dos Santos Klem received the Licentiate and M.S. degrees from São Paulo State University (UNESP), Presidente Prudente, Brazil, in 2015 and 2017, respectively, where he is currently pursuing the Ph.D. degree with the School of Technology and Sciences. His current research interests include printed electronics and energy storage devices.

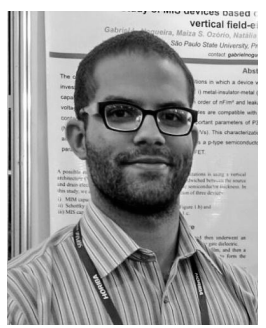

Gabriel Leonardo Nogueira received the Licentiate degree in physics and the M.S. degree from São Paulo State University (UNESP), Presidente Prudente, Brazil, in 2014, and 2016, respectively, where he is currently pursuing the Ph.D. degree with the School of Technology and Sciences. His current research interests include printed electronic and field-effect devices.

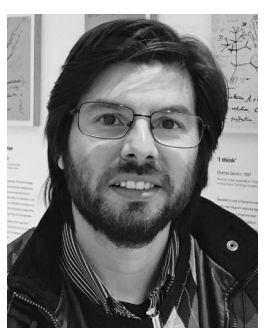

Tiago Carneiro Gomes received the master's degree in science and technology of materials from São Paulo State University (UNESP) in 2014, where he is currently pursuing the Ph.D. degree in science and technology of materials. His current research topics are oxide metal materials and organic semiconductors for flexible and printed devices.

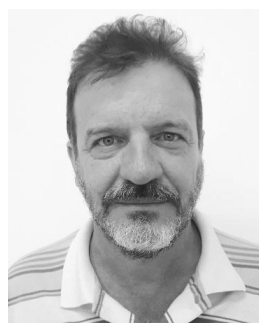

Neri Alves received the Ph.D. degree in applied sciences from the University of São Paulo, Brazil, in 1992. He is currently an Associate Professor with São Paulo State University (UNESP). His current research interests include organic electronic, printed electronics, and sensors 